\title{
AN ESSAY
}

oN

\section{THE PROXIMATE CAUSE}

\author{
OF THE DISEASE CALLed
}

\section{PHLEGMASIA DOLENS.}

\author{
By DAVID D. DAVIS, M.D.
}

Read May 6, 1823.

THE remarkable affection of the lower extremities incident to women during the period of their confinement in child-bed, which is now pretty generally known in this country under the designation of phlegmasia dolens, has not hitherto been the subject of accurate and sufficient pathological investigation. The facts to be detailed in the present communication are submitted to the society as a small and first contribution, to be followed, I trust, by others from other quarters, towards the developement of the proximate cause of that disease.

Up to the date of Case No. 1, that of Mrs. Caroline Dunn, to be presently related, I find suggested 
by authors, and admitted into books of nosology, four different theories upon this subject; all of them, it must be acknowledged, more or less plausibly derived from some of the known and obvious phenomena of the distressing malady.

As I hope to be able, in a great measure, to establish, on the evidence of morbid anatomy, the point of pathology now about to be proposed, I shall content myself with simply stating the substance of these doctrines; and refer gentlemen, who may be desirous of further information upon such matters, to the very able and elaborate essay on Phlegmasia Dolens, by my friend, Dr. Hull, of Manchester.

The first general idea on this subject deserving of notice, is that which was very modestly suggested by Mauriceau, in which he imputes the disease in question to metastasis of the lochia, or, to use his own words, " to a reflux determined to these parts" (the lower extremities) "of humours, which ought to be evacuated by the lochia."-Mauriceau, Traité des maladies des femmes grosses et de celles qui sont accouchées. 5eme edit. vol. i. p. 446.

The same doctrine, with some modifications, is adopted by Mesnard, who refers the swellings of the lower extremities of puerperal women to " suppression of their lochia, producing an over fulness 
of their blood-vessels, and a consequent arrest and coagulation of lymph in the parts affected."-Mesnard, Guide des Accoucheurs, Paris, 1743.

The second theory to account for the same phenomena, consists in a particular application to this subject of the celebrated doctrine of metastasis of milk-depôts de lait. This seems to have been first advanced by Puzos towards the middle of the last century, and was published, in the posthumous edition of his works, in $1759, i$. e. six years after his death. It was adopted by Levret, and made the subject of a communication from that gentleman to the Journal de Medicine of Paris, for the month of July in the same year. Since that period, it has maintained a brilliant reputation in the continental schools of medicine; where, indeed, it has assumed several varieties of forms to suit the plastic fancies of different writers who have received and supported it.

A third theory which has been formed on this subject, and which, like its predecessor, has been presented under several different modifications, is that of obstructions, or other morbid states of the lymphatic organs of the parts affected. With whom, and at what precise date, this notion originated, I am not able positively to state. The authors who first espoused it, in publications professedly on the subject, have been Mr. Charles White, 
of Manchester, and Mr. Charles Brandon Trye, of Gloucester; the former in 1784, and the latter in 1792.

Dr. Hull published his Essay on Phlegmasia Dolens in 1800. After criticising at some length, and with the ability which both his friends and his enemies have well known how to appreciate, the doctrinal speculations of his predecessors, he propounded a new theory of his own; which, to do it perfect justice, I shall take the liberty of stating in his own words.

"The proximate cause" (of phlegmasia dolens) " consists in an inflammatory affection, producing suddenly a considerable effusion of serum and coagulating lymph from the exhalants into the cellular membrane of the limb. The seat of the inflammation I believe to be in the muscles, cellular membrane, and inferior surface of the cutis. In some cases, perhaps, the inflammation may be communicated from those parts to the large blood-vessels, nerves, and the lymphatic vessels, and glands imbedded in them."

In the statement of this theory, we have two distinct propositions advarced : viz. 1st, that the disease is an inflammation presumed to have the effect of suddenly producing a considerable effusion of serum and lymph into the cellular membrane : 
and 2dly, that the muscles, cellular membrane, and inferior surface of the cutis, are essentially and originally the seat of such inflammation.

It is particularly worthy of remark, that this very capacious theory of a proximate cause of disease, so extensive in its primary operation as to require for its seat and subject-matter such a great variety of structure, is not professed, by its learned author, to be founded upon any evidence derived from examination after death of the parts affected. I am, indeed, fully aware, that no evidence of this kind existed at the date of Dr. Hull's publication; if, however, we except one most important case-a case to which the Doctor himself refers, though he is scarcely willing to consider it as a genuine example of phlegmasia dolens. The observation I allude to was originally published by J. Gottfrey Zinn, in 1753, in the second volume, p. 364, of The Commentaries of the Royal Society of Sciences at Gottingen.

As I see no sufficient reason for rejecting this solitary and long-neglected case; as it is given in a small compass, and with much apparent fidelity; and as it will be found to bear very closely upon what, I trust, will soon appear to be the true pathology of the disease under consideration, I shall take the liberty of quoting it. I shall adopt the version of the editors of the Medical Museum 
(vol. i. p. 335), excepting in one or two passages where the meaning of the original is not duly rendered.

\section{"OBSERVATION. \\ " AN CEDEMATOUS FOOT, FROM A COMPRESSURE OF THE CRURAL VEIN.}

"A woman, nearly thirty years of age, after a difficult labour, and in consequence of careless conduct, suffered much disturbance of her lochia. Her right leg was seized with an œdematous swelling, which extended from the groin to the heel, and enlarged the right labium pudendi. At the same time she was also seized with a loss of appetite.

" Every probable means afforded by the art of healing was tried to remove the swelling, but without success. Neither diaphoretics, nor purgatives, nor diuretics, gave any relief; and fomentations and frictions excited the most violent pain. An incision was made through the cutis of the thigh, that the water might be drained off by an issue; but only a few small drops were discharged by it. The serum, in the cellular membrane, assumed in some sort the nature of a tremulous gelatine; all the more fluid part of it being resorbed. At the end of two months the patient died asthmatic.

“ On dissecting the body, we found some of the 
inguinal glands schirrous, greatly enlarged, and surrounding the crural vein, by which its diameter was very much diminished."

In an analysis of this dissection, it is important to distinguish between the facts that are reported, and the opinion of the writer as to the order of their relation to each other as cause and effect. Thesimple facts of the case are enlargement and induration of the inguinal glands, and a great diminution of diameter of the crural vein. That this diminution of diameter in the vein was the effect of the compression presumed to have been made upon it by the enlarged and indurated glands, is to be received as a matter purely of opinion. In admitting, therefore, the fact of a diminution of diameter in the vein, we are by no means bound by the author's opinion as to the cause. On the contrary, it is my firm belief, that the actual cause of the asserted diminution of capacity in the vein, was the effect of a primary disease of the vessel itself; and that the inguinal glands had become enlarged and indurated in consequence of their immediate vicinity to the original seat of disease in the crural vein.

It is a matter of obvious inference from the perusal of the above case, though it is not expressly stated, that the interior of the vein was not made the subject of pathological research; otherwise, I am bound to presume that the author would have given us a description of a diseased condition of the 
constituent structure, as also of what he calls the " lumen" of the crural vein, which, I take for granted, he would have then and there discovered.

I have now to submit to the Society the results of my own opportunities for ascertaining the morbid changes productive of the phenomena of phlegmasia dolens; together with an important additional contribution from a good pathologist, and a much-esteemed surgeon in the country, $\mathrm{Mr}$. Henry Oldknow, of Nottingham.

In the developement of these facts, it will be my object to prove, or at least to attempt to prove, that the proximate cause of the disease called phlegmasia dolens, is a violent inflammation of one or more of the principal veins within and in the immediate neighbourhood of the pelvis, producing an increased thickness of their coats, the formation of false membranes on their internal surface, a gradual coagulation of their contents, and occasionally a destructive suppuration of their whole texture; in consequence of which, the diameters of the cavities of these important vessels become so greatly diminished, sometimes so totally obstructed as to be rendered mechanically incompetent to carry forward into their corresponding trunks the venous blood brought to them by their inferior contributory branches.

The following cases, accompanied by their se- 
veral dissections, are offered in solution of the problem here stated. They are given respectively in the order of their dates.

\section{CASE $I$.}

Caroline Dunn, æt. 21, of a weak constitution, was delivered of a male child on the 7th of February, 1817, after a severe labour of twenty-seven hours' duration. Some loss of blood was sustained both before and after the birth of the child. On account of the latter hæmorrhage the placenta was removed artificially by the introduction of the hand into the uterus.

On the following day the pulse was full and regular at 90. The tongue was white but moist, and there was a slight thirst. Pressure upon the abdomen occasioned no pain : but a soreness was felt in the vagina.

During the days immediately succeeding, the symptoms were moderate. On the 13th the case was reported as follows._- "Slight fever, pulse quick and full, bowels costive, tongue white and dry, labia pudendi inflamed, swelled, and odematous, some head-ache, respiration difficult, appetite bad, a copious yellow discharge from the vagina, having the consistence of cream, but without foetor." 
17th. The report was, " Better generally :-discharge much decreased, inflammation subsided, bowels well relieved, pulse regular at 86 , tongue natural in its appearance, thirst still great.

“ 21st. Much better :-sleep good, sat up out of bed for four hours.

“ 22nd. Better still :-complained of slight pain, like cramp, as the patient herself expressed it, in the left leg.

“26th. Worse : left leg and thigh much swollen; pain in the inguinal region, skin hot, no signs externally of inflammation, no pitting on pressure, bowels costive, slight cough, respiration difficult, pulse very quick and small, headache.

“Feb. 28th to March 2nd. No better:-leg pitted on pressure, countenance depressed, languor, giddiness at intervals, pulse 80 , freedom from pain, no appetite, bowels twice relieved.

“ 3d. Total insensibility :-limb equally swollen, countenance sunk, pale, and emaciated.

" 4th. Died at noon this day."

The above account is an abstract of the leading facts of the case taken from more copious notes 
which were furnished to me by the young gentleman, Mr. Francis Hunt, from Bath, at that time my pupil, who had attended the patient.

Not satisfied with any theory of the disease which up to the date of the unfortunate case just related had been promulgated, I sought the earliest opportunity of obtaining the husband's permission to inspect the body : and, feeling anxious that the report of any remarkable appearances which might present themselves should be entitled to the most perfect confidence of the profession, I made application to my friend, Mr. Lawrence, to undertake that duty, which he very kindly acceded to; and at my further request, he obligingly sent me by the next day's post, the following account of the appearances on dissection. "See Plate VI, Fig. I.

"Appearances observed on examining the Body of Caroline Dunn, on the 6th of March, 1817.Present Dr. Davis.

“The left lower extremity presented an uniform œdematous enlargement, without any external discoloration from the hip to the foot. This was found, on further examination, to proceed from the ordinary anasarcous effusion into the cellular substance. The inguinal glands were a little enlarged, as they usually are in a dropsical limb; but pale coloured, and free from the slightest sign of inflammation. The femoral vein from the ham up- 
wards, the external iliac and the common iliac veins as far as the junction of the latter, with the corresponding trunk of the right side, were distended and firmly plugged with what appeared externally a coagulum of blood. The femoral portion of the vein, slightly thickened in its coats, and of a deep red colour, was filled with a firm bloody coagulum closely adhering to the sides of the tube, so that it could not be drawn out. As the red colour of the vein might have been caused by the red clot every where in close contact with it, it cannot be deemed a proof of inflammation. The trunk of the profunda was distended in the same way as that of the femoral vein; but the saphena and its branches were empty and healthy. The substance filling the external iliac and common iliac portions of the vein was like the laminated coagulum of an aneurismal sac, at least with a very slight mixture of red particles. The tube was completely obstructed by this matter, more intimately connected to its surface than in the femoral vein ; adhering, indeed, as firmly as the coagulum does to any part of an old aneurismal sac. But in its centre there was a cavity containing about a tea-spoonful of a thick fluid of the consistence of pus of a light brownish red tint, and pultaceous appearance.

The uterus which had contracted to the usual degree at such a distance of time from delivery, its appendages and blood-vessels and the vagina 
were in a perfectly natural state. There was not the least appearance of vascular congestion about the organ, nor the slightest distension of any of its vessels. Its whole substance was on the contrary pale, and the vessels every where contracted and empty.

\&6 The state of the abdominal cavity and its con. tents was perfectly natural.

"c That the substance occupying the upper part of the venous trunk, and the fluid in its central cavity had been deposited there during life from inflammation of the vessel does not admit of doubt. I am also decidedly of opinion, in consequence of its firmness and close adhesion to the vein, that the red coagulum in the femoral vein was the result of a similar affection extending along the tube; and that the passage of blood through it in the whole tract submitted to examination, must have been completely obstructed before death.

$$
\text { "WM. Lawrence." }
$$

" College of Physicians."

The above case, after exciting considerable interest among my pupils and their immediate friends at the time of its occurrence, was made the subject of a public debate or conversation at the Bartholomew Society in the spring of the same year. I state this fact on the authority of Mr. Hunt and other students of that period; and I have no doubt vor. XII. G 6 
that it is a matter of perfectly distinct recollection with some gentlemen who are still in the habit of paying occasional visits to that justly celebrated hospital. Since that time, I have myself constantly made it the subject of discusion in my lectures as well as of conversation with my professional friends.

\section{CASE II.}

Mrs. C. a lady of a sanguineous irritable temperament, died suddenly in the midst of apparently high and perfect health, on the 20th of September, 1819 , in her sixth week after her second puerperal confinement. On the day after her delivery, she was seized with violent peritoneal inflammation, which, however, yielded, after a pretty smart conflict, to free and repeated bleedings, together with the application of between forty and fifty leeches to the abdomen. The febrile excitement was nevertheless not completely subdued by these measures; and in about ten days afterwards, I was apprized by my friend Mr. Anderson, of Fleet-street, to whom I was indebted for much substantial assistance in the management of this case, that our patient had made complaint of a deep-seated pain in the groin and along the tract of the great vessels of the thigh. On first inspecting the limb, I found it considerably swelled and exquisitely painful. By the prompt application of leeches to the groin and 
interior of the thigh, and afterwards by blistering extensively the same surfaces, this new inflammation was speedily reduced, and, in about a week from its commencement, the swelling had entirely subsided, and the patient had recovered the full power of contracting and extending the extremity without suffering pain. From this period she convalesced rapidly and satisfactorily. Her death took place instantaneously, whilst in the act of changing the recumbent for a sitting position, in the expression of a little merriment at the expense of something ludicrous which her waiting woman had said to her, and in about an hour after the enjoyment of an unusually full dinner.

Dissection._- In the examination of the late Mrs. C. in the presence of Dr. Davis, Mr. George Anderson, and John C. Taunton, the following circumstances were noticed:-

"The external appearance of the body was healthy and natural, no apparent loss of flesh nor strength having been sustained.

"The external surface of the thoracic viscera was natural, the lungs were healthy, the pericandium and large blood-vessels were all perfectly natural.

"In the abdomen, adhesions between the viscera and the parietes were observed, chiefly at the upper part of the cavity. These were the consequence 
of previous inflammation, which had completely subsided, and neither it nor its results could have had any agency in producing the death. The blood vessels in the upper part of the small intestines were a little surcharged with blood. The stomach, with its contents, was natural. The gallbladder contained a small quantity of bile. The bile-ducts were free. The liver, spleen, pancreas, and kidneys, were all healthy. The vessels, both arteries and veins, were quite free from disease. The uterus and its appendages, together with all the pelvic viscera, were natural and healthy.

"John Taunton, Surgeon.

"September 21, 1819."

I have to regret that Mr. Taunton, after examining the uterus and its-appendages, with which he concludes the above description, was obliged to leave us somewhat hastily, on account of another urgent claim on his time. Mr. George Anderson and Mr. John C. Taunton were, however, kind enough, at my request, to undertake the examination of the iliac veins. It is to them that $I$ am indebted for the preparation, No. 2. It forms a part of the left external iliac vein, including about half an inch of the upper portion of its corresponding femoral vein. That vessel was found strongly attached by adhesions of its cellular coat to the parts forming its natural bed. Its parietes still retained a morbid thickness, and its internal tunic was studded in several places with deposits of ad- 
herent lymph. The portion most remarkable for this incrustation, and otherwise most diseased, was the part of the vein immediately under Poupart's ligament. The appearance of that part is yet well preserved in the preparation, and forms the rough scabrous inferior portion of it. The tube of the vessel was still manifestly pervious, though it had suffered a diminution of capacity, amounting to, perhaps, one-half of its natural diameter. The inguinal glands were not diseased. The right iliac vein was in a perfectly healthy state. Plate VI. Fig. 2.

For the next case, I am indebted to the kindness and liberality of Mr. Oldknow, of Nottingham.

\section{CASE III.}

“ Jane Elliott was delivered in September, 1820, having an easy and natural labour. Until the 20th day after the delivery she was doing well; on that day she was seized with a violent purging*, for which astringents were given with success; but the pulse continued quick, and she had considerable fever. On the 30th day, the purging returned, and the left lower extremity became swollen and painful, with considerable increase of fever. She died on the 34 th day.

* Diarrhœa was prevalent at the time. 
Dissectron._- " On examining the swollen limb the day after her death, I found the femoral vein, one-third down the thigh and all the iliac veins, much enlarged, and containing adherent layers of coagulated blood, similar to that found in aneurismal sacs, together with a sort of grumous fluid of a brown colour, more or less mixed with air, and almost obliterating the venous canals. The same appearances, but in a much less degree, extended along the cava as far as the entrance of the renal veins. The coats of the veins were highly inflamed, and intimately attached to the surrounding parts. The absorbent vessels and glands were slightly enlarged as high as the lumbar regions, but not otherwise affected. The uterus had regained nearly its natural size."

In connexion with the preparation, No.3, which accompanies the above important dissection, I feel it a duty to give insertion to a part of a letter which I had the honour of receiving from Mr. Oldknow, at the same time with the specimen itself.

CG MY DEAR SIR,

"I SEND you the preparation; but I regret that it so imperfect. For owing to having allowed it to macerate in water too long, the extent of the deposition is not seen; it having separated from the internal surface of the vein in flakes of various sizes. The principal one remaining (and which is seen in the preparation, though in a very imperfect 
state) is the inguinal and contiguous portion of the external iliac veins. But the description which you possess, the accuracy of which I vouch for, must supply the deficiency. The small pieces of bougie are placed in the internal iliac artery where it passes over the vein.

"I remain, my dear sir, "Yours, truly, "H. Oldknow."

See Plate VII.

\section{CASE IV.}

Mrs. L...... a lady' of a delicate constitution, and of a very irritable habit, was delivered of her fifth child on the 2nd day of July, 1821. She had been subject to feverish affections in several of her former confinements. On this occasion she was doing well until the seventh day after her delivery, when she was taken out of bed and placed upon a sofa, between the fire and a large loosely hung window. In this situation she was seized with a violent rigor. During the gradual developement of the succeeding hot fit, she experienced a pain of the left side of the chest, which increased rapidly in intensity. She was freely bled before I saw her, without experiencing adequate relief. She was afterwards bled, leeched, and blistered. The pain of the chest was then in a great measure subdued; 
but this flattering advantage was not accompanied by any material reduction of the attendant fever. The pulse on the contrary continued quick, and the general distress and restlessness were so little mitigated as to call for much sympathy, and to excite serious alarm. In the evening of the same day, unequivocal symptoms of phlegmasia dolens declared themselves. The patient died on the 23d of the month.

Dissection.-Present, Dr. Sims and Mr. Macdonald, who had assisted me in the treatment of the case ; and Dr. Courthope Sims.

The pleura costalis of the left thoracic cavity was found slightly inflamed, and litred in two or three places with a delicate tunic of lymph. There was also an effusion of about six ounces of a nearly transparent serosity into the cavity itself. The lung of the same side was of a dark red hue. The heart and pericardium presented no appearance of disease. The right cavity of the chest and its contents were also healthy.

All the surfaces and viscera of the abdomen were apparently in a perfectly sound state. The left lower extremity, from the hip to the toe, was considerably but not greatly enlarged, and there was an evident fulness of the labium pudendi of the same side. The iliac veins of both sides were unusually turgid with blood; but presenting no other 
external manifestation of disease. They were entirely free from attachments to the contiguous parts. The inguinal glands were certainly not diseased, nor even visibly enlarged. On making a careful incision into the left external iliac, it was found to contain a coagulum of blood of firm consistence; but not at that part adherent to the internal surface of the vein. Upon examining, however, the common iliac portion of the vessel, adhesion of the same column of coagulum had obviously taken place; an appearance which is now distinctly to be seen in the preparation. (See preparation No. 4). The left internal iliac was greatly inflamed, and its diameter was so much contracted by the morbid thickening of its parietes, that it was rendered almost impervious. The right iliac vein, including both its common and external portions, was distended with a similar coagulum; or rather the same column of coagulum was prolonged over the angle of their common junction with the vena cava from one iliac to the other. In this interesting fact of the dissection we cannot fail to recognize, had the poor patient's life been preserved, the materials in actual preparation for a similar affection of the right limb. Plate VIII.

As a sequel to the above cases I may be permitted to add a short abstract, just published, in the Medical Repository for the present month, of two pathological histories, one on the same, and 
the other on an analogous subject, given in the Journal de Physiologie of Paris for last January. It will be seen that the phenomena of the last case, which was the only case of true puerperal phlegmasia dolens, were attributed to a circumstance probably purely adventitious.

"M. Bouillaud has observed in several cases of infiltration of the lower extremities, the venous trunks entering the pelvis quite impermeable and filled with a concrete and organized coagulum.

"In the case of a female, who died from chronic peritonitis and schirrus of both ovaria, with immense infiltration of both the lower limbs, the veins near their opening into the cava were in a manner obliterated by the pressure of the diseased ovaria, and completely filled from their junction with the cava with a carnified friable coagulum.

"In another female, aged 38, who soon after her confinement had great œdema of the lower extremity, and who soon afterwards died, on dissection, the veins of that limb were found to be filled with a similar coagulum to that of the former case, which extended to the vena cava. The other veins were in their usual state. The iliac portion of the colon was distended with a large mass of indurated freces, which pressed on the adjoining veins, and gave rise to their impervious state and to the infil- 
tration of the limb. These observations were made in a Parisian hospital." Jan. 1823. Journal de Physiologie.

I shall conclude with a few general observations in application of the above facts to the known phenomena of the disease.

The primary seat of pain, on the accession of phlegmasia dolens, is the precise locality of the inflammation and other diseased affections of the veins of the pelvis, as observed upon dissection in the above cases. See Levret. L'Art des Accouchemens. Des Infiltrations laiteuses, troisième édition, p. 176.

"The first complaint," says Mr. Trye, "made by the patient, in every case which $I$ have been acquainted with, was that of stiffness or soreness, in one of the lateral regions of the lower belly, and pain in moving or turning about the body." "Upon examination, a fulness and hardness in the affected iliac region may be perceived, and for the most part a circumscribed tumour. A weakness of the thigh and leg of the same side, and in most cases an œdematous swelling of them came on, but not always. The affection of the extremity has not been complained of for two or three days after the coming on of the pain in the flank." Trye's Essay, p. 6. 
Mr. White, in his general description of this disease, observes that, " in about twelve or fifteen days after delivery, the patient is seized with great pain in the groin of one side.".........." This part soon becomes affected with swelling and tension, which extend to the labium pudendi of the same side only, and down the inside of the thigh, to the ham, the leg, the foot, and the whole limb."....... "The pain in the groin is generally preceded by a pain in the small of the back, and sometimes by a pain in the bottom of the belly on the same side." White's Enquiry, p. 7.

Dr. Hull states, " that the complaint generally takes place on one side only at first, and the part where it commences is various; but it most commonly begins in the lumbar, hypogastric, or inguinal region on one side, or in the hip, or top of the thigh and corresponding labium pudendi." In a note, it is remarked by the same author, that " when it begins in the lumbar region, it appears to be propagated along the psoadic and iliac muscles to the groin, and resembles a severe case of lumbago. When it affects the hip first, it appears under the form of a violent ischias or sciatica." Hull's Essay on Phlegmasia Dolens, p. 134.

I have myself met with some cases, where the pain first and most complained of has been in the calf of the leg, or some other inferior part of the affected extremity; but in all such cases I have also found, that, upon application of the gentlest pressure to 
the corresponding groin and iliac region, the patient has instantly flinched from the touch.

The swelling which succeeds to and accompanies the pain in phlegmasia dolens is first perceived in the groin and adjacent parts, and observes a regularly progressive course downwards from the pelvis to the toes. This fact is mentioned by almost all the writers on the disease, as an essential part of its history; and by several of the nosologists, as a pathognomonic symptom of it.

The principal veins of the pelvis are necessarily exposed to great pressure from the uterus during the latter months of gestation. A well-known effect of this pressure is a varicose dilatation of the superficial veins, together with œdema of the lower extremities ; - a state of the limbs indicative, in the opinion of practical writers, of a predisposition to phlegmasia dolens. Practitioners in midwifery are often consulted at those periods, on account of distressing pains and pinchings, which are referred to the small of the back, iliac regions, and groins, and which no doubt are occasioned by pressure of the distended uterus upon the parts lining and corresponding with the brim of the pelvis.

A striking evidence of organic injury sustained by the great veins within the pelvis, from pressure and distension during gestation, is recorded in the first volume of the London Medical Repository, p. 456, in a paper entitled, ' Case of Rupture of the 
internal iliac Vein during Pregnancy.' The subject of the case was in the ninth month of her sixth pregnancy. Her former children had been unusually large, one of them having weighed eleven pounds. In several of her former pregnancies she had complained of pain in the right iliac region, which had been greatly relieved by bleeding. But on account of her absence from town till within a few weeks of her expected delivery, the same precautionary measure was unfortunately not adopted on this occasion. On the night of her death, she was suddenly awoke by a violent and deep-seated pain in the right groin and extending towards the hips. "An extraordinary sensation of coldness ensued, accompanied with a sense of fulness and distension of the abdomen, and pressure on the thorax, occasioning very laborious breathing." Then follows an enumeration of the moribund symptoms, such as usually supervene upon the rupture of great vessels situated within the trunk of the body. "The body was opened by Sir William Blizard. Upon dividing the integuments, the whole of the abdomen not occupied by the gravid uterus was filled with coagulum. The uterus was in its natural situation, but was of immense bulk and weight from a fœetus of an unusual size. The hæmorrhage did not proceed from any of the vessels of the uterus; but a rupture of the internal iliac vein was discovered, through which the refluent blood had passed into the cavity of the abdomen."

It is easy to conceive, that diseased affections of 
contiguous parts may operate as causes of irritation upon the great veins of the pelvis, and, by quickly injuring their natural and healthy organization, become productive of phenomena similar to those of phlegmasia dolens. M. Bouillaud's first case is to be attributed probably to an influence of this kind. A well detailed example, illustrative of the same important fact is recorded in the Journal der Practischen Heilkunde, of Hufeland, for June, 1820. An instance of the same fact is recorded by Morgagni, letter 56, art. 10. A mild case of phlegmasia dolens succeeded to rupture of the uterus in a patient of Mr. Hugo's, of Crediton, who recovered after that tremendous accident was published by that gentleman in the London Medical and Physical Journal, vol. xix. p. 212.

Since writing the above, my friend, Dr. Birkbeck, has kindly sent me some particulars of a case of swelled lower extremity in a lady who had not been parturient for many years, occasioned, as it was supposed, by a diseased state of some of the organs within the pelvis, in which he could distinctly trace the femoral vein in its usual course along the superior and interior part of the thigh into the groin, greatly enlarged and indurated. "The regular form of it was interrupted by indentations at intervals, appearing to coincide with the position of the valves."

But it appears probable that such diseased affec- 
tions of contiguous parts, in order to become productive of the peculiar swelling and other symptoms of phlegmasia dolens, must be propagated to the veins concerned somewhat suddenly, after the manner of acute diseases, and also extensively, so as to impede their circulation almost perfectly and at once; as otherwise we might presume that the process of opening or widening new channels by the accommodating operations of the principle of anastomosis, would keep pace with the obstructions and obliterations of the old ones. Hence we have some examples recorded of obliteration of the iliac veins, from causes very slowly and gradually applied, where no symptoms indicative of obstruction in the venous circulation had presented themselves during the life of the patient. See Hodgson's Treatise on the Diseases of Arteries and Veins, p. 530.

It does not seem necessary to extend this mode of explanation to account for the absence of some of the peculiar symptoms of the swelled leg in the case of obliterated vena cava, and iliac veins, published some years ago, by the late Mr. Wilson, in the Transactions of a. Society for the Improvement of Medical and Chirurgical Knowledge, vol. iii.,p. 70 ; inasmuch as the original locality of the disease in that case, which probably was the inferior portion of the vena cava, is not presumed, in the present communication, to be the proximate seat of the obstruction to the venous circulation in phlegmasia 
dolens. On the other hand, we have no evidence that the patient had not been the subject of phlegmasia dolens during the first weeks of her confinement; though, upon the whole, it seems more probable that the intumescence, which might be expected to have presented itself, as the result of the obstruction to the circulation in her particular case, had only affected the parts in the immediate neighbourhood of the pelvis.

It is at present perfectly well known that diseases of veins are often attended by very formidable symptoms, and that occasionally they prove fatal in their events. Of the correctness of this statement abundance of evidence is furnished by Mr. Travers in his valuable Essay lately published " on Wounds and Ligatures of Veins." See also a good paper containing some new facts in review of Mr. Travers's Essay, signed " BREscher," which has recently appeared in the second volume of the Journal Complimentaire du Dictionnaire des Sciences Medicales. Many similar testimonies are likewise to be found in the pathological works of Hunter, Abernethy, Wilson, and Hodgson.

Phlegmasia dolens is a disease which almost always occurs in an excited state of the organs of the circulation; and when that is not the case, it is known to take place during a period of predisponency to inflammatory action of the great bloodvessels within the pelvis, and their immediate rami- 
fications. Hence its occurrence after labours and miscarriages, before the perfect re-establishment of the balance of the circulation; and at those times, more particularly, after profuse hæmorrhages, or during phlegmasiæ of other parts ; or from the application of occasional causes of whatever kind which may have the effect of exciting a febrile disturbance in the system.

All the veins liable to much pressure, or to enlargement of diameter during pregnancy, appear to be more or less predisposed to inflammation upon the sudden removal of those agencies, by the consummation of the act of parturition. Hence the predisposition, especially to hysteritis and peritonitis during the first week after labour, and to the swelled leg, and to mammary abscesses at a more advanced period. See Wilson's Lectures on the Blood, \&c., published in 1819. Lect. 15, pp. 414, 415.

It is a part of the history of phlegmasia dolens, that it is seldom known to attack the same extremity more than once. The explanation of this curious fact will possibly be found in the probability, much to be presumed upon, that the entire system of great veins within the pelvis is, in such cases, totally obstructed by the disease, converted into ligamentous structure, and therefore rendered unsusceptible of a similar disease in future. But I am positive on the evidence of several cases, which 
I have myself seen or examined, as well as on that of published authorities of unquestionable authenticity, that there are occasional exceptions to the rule. See Medico-Chirurgical Journal, vol. ii. p. 497.

Such exceptions, which, after all, are extremely rare, are probably to be attributed to the disease in the first instance proving incompetent to consummate perfectly its destructive work, so as to render the veins affected by it totally impervious; or, perhaps, still more probably, to some of the veins within the pelvis escaping the first attack of the disease altogether, as in the case of Caroline Dunn, in the description of which Mr. Lawrence states, that the internal iliac vein, and vena saphena were found entirely free from disease.

If the proximate cause of phlegmasia dolens, presumed in this paper, be indeed the true one, it should follow as an inevitable consequence, that after an extremity shall have been once thoroughly affected by the disease, the business of the circulation would have to be carried on in future by the establishment of an extensive system of anastomosis. Accordingly, I take upon myself to assert, that, in a very large proportion of cases, which by the favour of many friends I have had opportunities of examining, where the inferior extremities had been so affected, I have had the satisfaction of observing that important confirmatory 
result. The superficial veins, sometimes the very finest cutaneous veins, are to be seen, in many such cases, much enlarged, and, clustering together into extensive varices, increasing proportionally in dimension as they ascend high on the limb, to ramify in still bolder paintings on the hips and abdominal regions.

But I must acknowledge that these appearances, though to be easily recognized in a great majority of cases, have not invariably presented themselves. The more particular explanation of that fact I must leave to practical anatomists, and to future opportunities of observation. I would remark generally, that it must depend on peculiar varieties in the distribution of the new circulation. See the interesting dissections of Dr. Baillie and Mr. Wilson, in cases of obliteration of the vena cava, and other great veins within the trunk of the body. Transactions of a Society for the Improvement of Medical and Chirurgical Knowledge, vol. i. p. 127. plate 5.; and vol. iii. p. 65-70. and 80.

The.tedious process for the establishment of a new and circuitous circulation, is an evil of great and almost incalculable magnitude. It is painful throughout its whole duration, and for the first fortnight it is generally attended with extreme suffering. The time which it requires for its full accomplishment, may be said to vary from five 
weeks to about five months; and when the latter period is occupied, the patient seldom or never afterwards recovers a perfectly good state of health.

From a due consideration of these facts, it follows, that our main indication of treatment should have for its object the speedy subduction of the inflammation in the iliac veins; so as to guard against the more immediately dangerous terminations of inflammation in great veins, to prevent obliteration of their cavities, and even, if possible, any material reduction of their diameters; and thus to supersede the necessity for instituting the anastomosing process for the establishment of a circuitous circulation.

The proximate cause of phlegmasia dolens having been proved to consist in a violent and destructive inflammation of the iliac veins and their contributory branches, including in some cases the inferior portion of the vena cava; whilst at the same time the disorder itself is known to be ushered in and accompanied by unequivocal symptoms of pyrexia; it might very naturally occur that general bleeding would best and most speedily and certainly answer the indication proposed. In every case, however, where I have been a party to the adoption of such practice, or where I have had the opportunity of observing the effects of it in the practice of others, I feel it my duty to-state, that it has completely disappointed expectation. 
I select the following example from among several other cases in my possession, similarly treated, to represent my uniform experience of the ineffciency, to say the least of it, and it is all I wish at present to insist upon, of general bleeding in this disease. The subject of the history was a patient of one of our lying-in hospitals, and the gentleman whose name is subscribed to it, and who kindly drew it up at my request, was one of my best informed pupils at the period of its date.

"An unmarried woman, aged thirty, was, on the 5th of October, 1818, delivered of her first child, after an easy natural labour. On the third day after her delivery, a cathartic medicine was exhibited, which operated satisfactorily. The following evening (fourth day), she complained of severe pain in each groin, attended by headache and general irritation. The skin was now hot and dry, the tongue moist and whitish. The pulse 110, and rather full. The mammæ turgid with milk : the lochia regular. The headache had come on in the morning, accompanied by a pain in the right knee. In the afternoon, some slight shivering had supervened, to which, in a short time, the heat and inguinal pains had succeeded. These pains were circumscribed, and seated in the line of the iliac vessels, about two inches above Poupart's ligament. They were constant, acute, and much increased on pressure, but the integuments covering the parts were in no degree tender, nor was 
there any tumour or hardness to be felt. At times the pain extended down the thigh, shooting with severity into the calf of the leg, and sometimes stretching even to the toes. There was now no marked affection of either knee, but the pain on the right side was much more acute than that felt on the other. Twenty ounces of blood were immediately drawn from the arm, and an enema and brisk cathartic administered without delay. 5th day.-During the night, the cathartic operated well, and the morning found the patient cool, and her fever much reduced. But as the inguinal pains were still severe, ten ounces more of blood were taken from the arm, and small doses of sulphate of magnesia were exhibited at intervals during the day. 6th day.-The disease, though much reduced by these evacuations, was not, however, as yet subdued. Cupping-glasses, \&c. were therefore applied, first to the lower belly, and then to the loins; but though repeated attempts were made, and by different hands not unaccustomed to this duty, no blood could be thus obtained, nor could any satisfactory reason be assigned for this circumstance. Eighteen leeches were therefore substituted, which acted well. A blister was then applied to the right groin, and the saline laxatives continued as before. On the following day (the 7th) there was little complaint, but of debility; nor did any untoward circumstance, after this, retard the progress of the patient's recovery.

“ Nov. 12, 1818."

$$
\text { F. A. Macann, M.D.” }
$$


It is worthy of remark, that the subject of the above case was an unmarried woman who had had an easy labour, who had sustained no hæmorrhage, nor been exposed to any other causes of exhaustion. It is therefore to be presumed, that she could bear, with perfect impunity, the general bleedings which were had recourse to. But, at the same time, we may observe, that the disease was by no means subdued by them. On the contrary, we are distinctly informed, that the severe pain in the right iliac region was not removed until after the application of the leeches and the blister. I am not, indeed, acquainted with a single case among a great number which have been recorded since the time of Puzos, where the disease has been cut short at its commencement; or where, in other words, the establishment of the circuitous circulation has been prevented by general bleeding alone. I must however own, that the practice in question has not been without some very sincere and eager advocates. I beg to submit the following testimony in its favour to the deliberate estimate of the Society. " Although I am convinced," observes the celebrated Puzos, "of the necessity of bleedings in these sudden depôts, which are attended by fever, and which may be regarded as forming the commencement of an acute and inflammatory malady; nevertheless, I do not pretend that this method of cure which I propose is infallible. I have had the misfortune of losing more than one patient, in spite of all the bleedings that I could practise : not that the bleed- 
ings had been contra-indicated, but that they had been insufficient for such cases__"Puzos. Mémoire sur les dépôts laiteux, p. 355.

Phlegmasia dolens frequently occurs in exhausted states of the constitution, from hæmorrhages and long protracted fevers; where general bleeding could not be had recourse to without incurring extreme hazard. Add to this the important fact, that it is in the nature of the disease itself to rob the general system of a large proportion of its blood by locking it up in the affected extremity, so as to make it so far unavailable for the ordinary purposes of the circulation. Hence the palpitations, distressing faintings, and other symptoms of extreme debility, which usually supervene during the intumescence of the limb; an assemblage of symptoms, which, it is to be presumed, we have now good reason to attribute to the struggles of the heart, in its state of sudden privation, to carry on the business of life, and to restore the lost balance of the circulation.

Seeing, then, that general bleeding is so decidedly objectionable, both in theory and practice; I am happy to have it in my power to assure the Society, that the great indication of treatment in this disease as already proposed; viz. the speedy resolution of the inflammation in the iliac veins, is to be secured in almost every case (I have not seen an exception) by early and decisive local treat- 
ment. The blood to be abstracted should, accordingly, be all taken from the immediate neighbourhood of the part primarily affected. From the excessive tenderness of the parts concerned, leeches are the only operators to be depended upon in these cases. Of these a dozen or a dozen and a half should be forthwith applied to the groin, to the affected iliac region, and to the interior and superior part of the thigh. If this be done before any very obvious accumulation of blood in the limb shall have taken place, it will generally put down the threatened mischief at once. In the event of our first success proving incomplete, a large blister should be applied to the groin and parts adjacent, both above and below. These measures are to be varied and repeated according to the particular circumstances of the case. See Trye's Essay, p. $12,8 x$.

During the progress of the swelling, which is invariably accompanied by the evolution of much heat, the limb should be cooled, and afterwards kept at a low temperature by evaporating lotions and free and constant exposure to the action of the atmosphere. I attach much importance to this practice.

Some practitioners have placed great reliance upon the action of fomentations in phlegmasia dolens: and I can, indeed, suppose, that in very mild forms of it, they may be of some service. I do not 
however remember, that I have ever myself recommended them until after the previous application of leeches; and then chiefly for the purpose of promoting a free discharge of blood from the part. I have known more than one instance of this disease being threatened, and presenting very distinctly some of its peculiar symptoms, and afterwards subsiding almost entirely of its own accord. It is only in such cases that I should be disposed to place exclusive dependence. upon the action of fomentations. For a very strong testimony, however, in favour of fomentations, see the fifth volume of the London Medical Journal, p. 95.

An esteemed friend has lately suggested to me, that the peculiar swelling incident to this malady might possibly be moderated by gentle bandaging. The hint is particularly worthy of consideration, as it appears to be in perfect accordance with the best known laws of the circulation.

I am not aware that I have ever derived any substantial advantage from the exhibition of antimonials. When it has been my object to reduce arterial action, and when I have felt any doubt as to the permanent efficacy of the measures already recommended, or met with cases of more than ordinary obstinacy, I have of late years had recourse to the use of digitalis in full and frequent doses; viz. in doses of two grains of the powder (Battley's preparation) every two, or, at furthest, every 
three hours. My experience of this mode of exhibiting the digitalis in acute diseases, enables me to state, with confidence, that it may be safely administered to adults at such intervals and in such quantities, until the patient shall have taken from twenty-five to thirty grains of it. It should then be proceeded with more slowly, until some one or more of its peculiar effects on the nervous system, or the circulation, be produced, when it should be immediately suspended; to be again resumed or not, according to circumstances. It will generally be an advantage to keep the circulation under its controul for several weeks, as an insurance against the accession of the disease in the other extremity. I need not observe that the foxglove is a potent drug, and that it requires much caution and constant watching in its exhibition. I generally combine it with a small quantity of the blue pill; which I think prevents it, in a great measure, from nauseating the stomach. I do not approve of the use of active purgatives in this disease. The bowels, of course, should be kept moderately open, as indeed should all other important functions of the system be placed under due and well-balanced regulation.

29, George Street, Hanover Square, May 6th, 1823. 
Explanation of the Plates illustrating the preceding Paper.

\section{PLATE VI. Fig. 1.}

CARoLINE DUNN's CASE.

$a$. Shews the superior part of the common and external iliac vein extremely diseased, and charged with a brownish purulent looking fluid obstructing its cavity.

b. The cavity of the accompanying artery having no appearance of disease.

c. The firm coagulum filling up the entire cavity of the femoral vein.

d. The same coagulum produced into contributory branches.

Fig. 2.

CASE OF MRS. C.

$f$. Deposits and incrustations of blood in a coagulated state. The inferior part of the interior surface of the diseased vessel very rough and scabrous from this cause.

g. Valves.

\section{PLATE VII.}

CASE OF MR. OLDKNOW'S PATIENT.

a. Adherent masses of coagulated blood nearly co-extensive with the internal surface of the whole portion of the left iliac and femoral 
vein. The same appearance in a less degree extending along the vena cava as far as the entrance of the renal veins.

$b$. The internal iliac artery passing over the vein to distribute itself upon the organs within the pelvis. No coagulum nor false membrane is to be seen attached to the internal surface of this artery.

c. A part of the vein without attachment of coagulum, and shewing valvular structure.

\section{PLATE VIII.}

CASE OF MRS. L.

a. Plug of coagulum in morbid adherence to the internal surface of the common iliac vein.

$b$. The left internal iliac vein turned on one side to shew the diseased structure of its internal surface. 
Plate VA

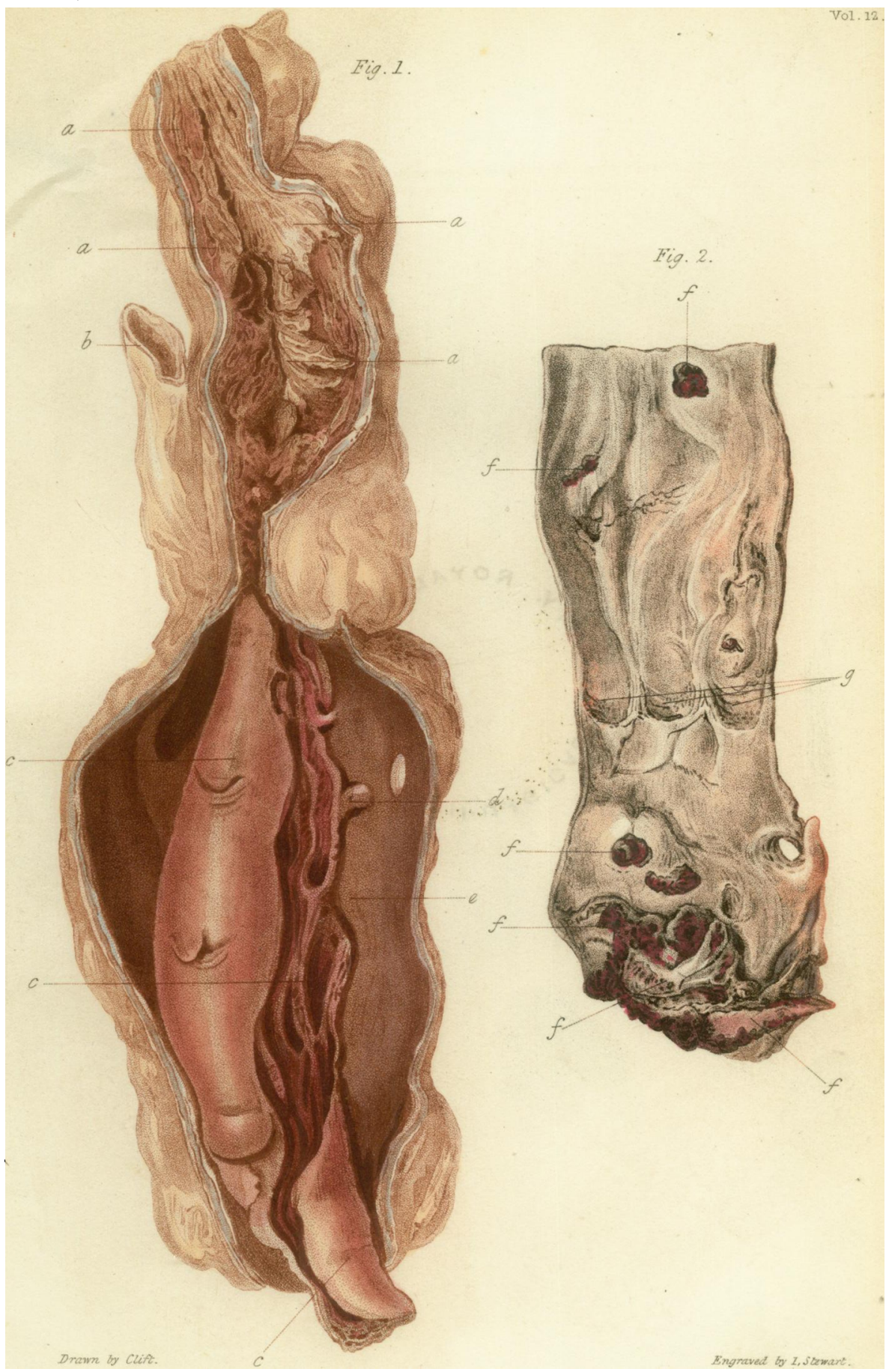




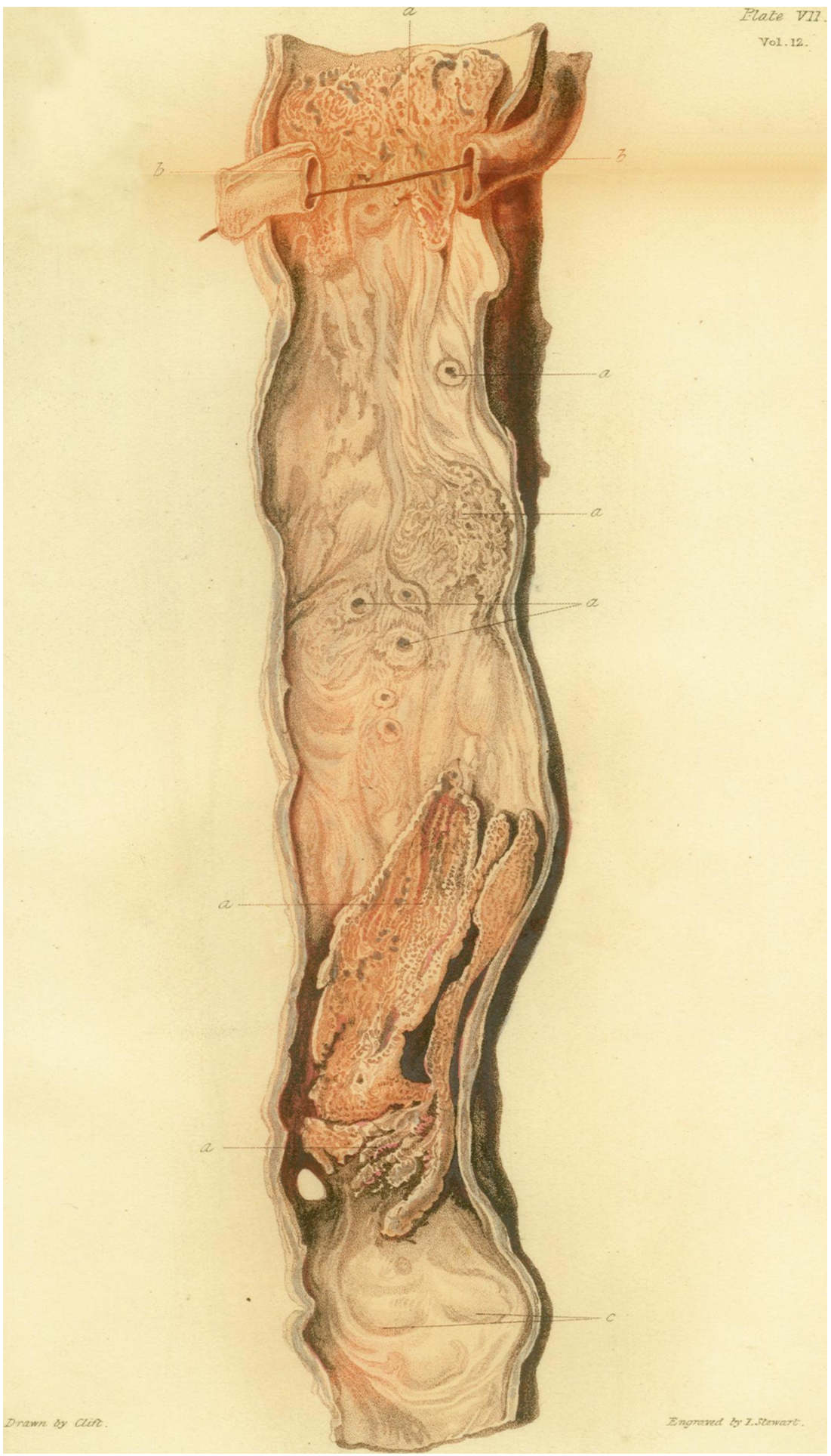




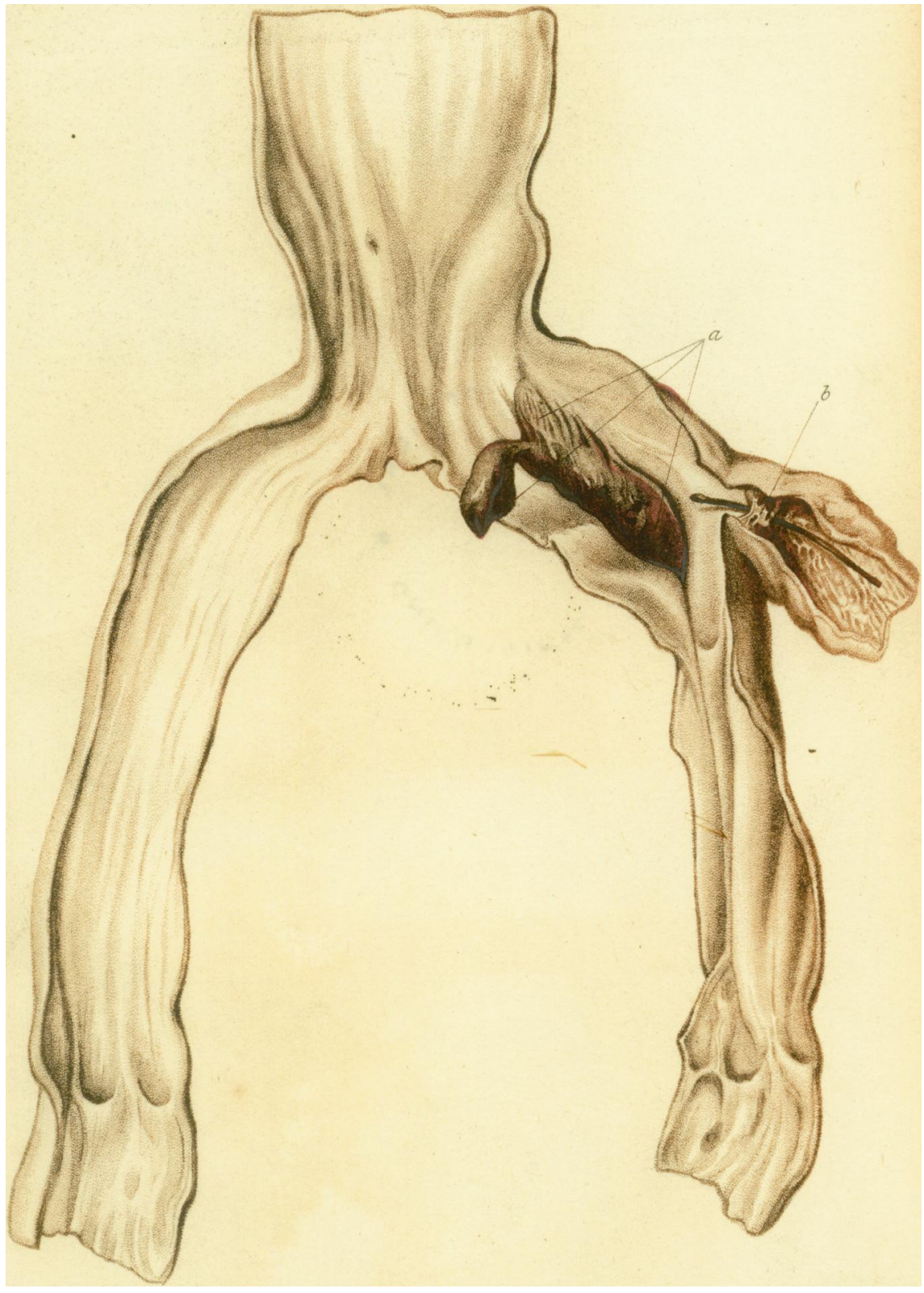

Drawn by Clift. 